\title{
Correction to: A Two-Layer Computational Model for Discrimination of Enhancer and Their Types Using Hybrid Features Space of Pseudo K-Tuple Nucleotide Composition
}

\author{
Muhammad Tahir $^{1} \cdot$ Maqsood Hayat $^{1} \cdot$ Sher Afzal Khan ${ }^{1}$
}

Published online: 5 December 2017

(C) King Fahd University of Petroleum \& Minerals 2017

\section{Correction to: Arab J Sci Eng}

https://doi.org/10.1007/s13369-017-2818-2

The original version of this article unfortunately contained a mistake. One character was missing in the title, which was therefore incorrectly given as "A Two-Layer Computational Model for Discrimination of Enhancer and Their Types Using Hybrid Features Pace of Pseudo K-Tuple Nucleotide Composition".

The correct version of the title is given above.

The original article can be found online at https://doi.org/10.1007/ s13369-017-2818-2.

Maqsood Hayat m.hayat@awkum.edu.pk

1 Department of Computer Science, Abdul Wali Khan University Mardan, Mardan, KP, Pakistan 